\title{
IL6-R blocking with tocilizumab in critically ill patients with hemophagocytic syndrome
}

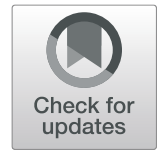

\author{
Etienne Dufranc ${ }^{1}$, Arnaud Del Bello ${ }^{1,2}$, Julie Belliere ${ }^{1,3,4}$, Nassim Kamar ${ }^{1,2,3}$, Stanislas Faguer ${ }^{1,3,4^{*}}$ on behalf of the \\ TAIDI (Toulouse Acquired Immune Deficiency and Infection) study group
}

Keywords: IL-6, Tocilizumab, Hemophagocytic lymphohistiocytosis, HLH

To the Editor,

Hemophagocytic lymphohistiocytosis (HLH) is a rare life-threatening hematological disorder characterized by uncontrolled activation of $\mathrm{CD}^{+} \mathrm{T}$ cells and NK cells, cytokine storm (including overproduction of interleukine6 (IL6)), and uncontrolled hemophagocytosis leading to severe organ dysfunction [1]. Several causes of HLH have been identified, including infection, cancer, drugs, and autoimmune diseases [1]. Diagnosis of HLH is challenging, and the $H$-score may help to better identify patients with reactive HLH [2].

A combination of dexamethasone, etoposide, and treatment of the underlying cause is the cornerstone of treatment for severe forms of HLH [1]. Because some patients may develop refractory or relapsing $\mathrm{HLH}$, alternative treatments targeting specific immune pathways or cytokine signaling have been tested [1]. These approaches also aim to avoid long-lasting etoposide-induced neutropenia in patients with bone marrow failure or after transplantation.

Tocilizumab, a monoclonal antibody targeting the receptor of IL6, fully reverses the multi-organ failure and the cytokine profile of the CAR-T cell-induced cytokinerelease syndrome [3]. This prompted some groups including ours to treat severe HLH secondary to acute autoimmune disease with tocilizumab [4]. Targeting one of the major cytokines that orchestrate the cytokine

\footnotetext{
* Correspondence: stanislas.faguer@inserm.fr

'Département de Néphrologie et Transplantation d'organes, Centre

Hospitalier Universitaire de Toulouse, F-31000 Toulouse, France

${ }^{3}$ Université Paul Sabatier - Toulouse 3, F-31000 Toulouse, France

Full list of author information is available at the end of the article
}

storm may be an alternative to etoposide in patients with HLH not related to hematological malignancies.

In the herein study, we reviewed the outcomes of nine critically ill patients who received tocilizumab to treat HLH (Table 1). Eight of them received at least one organ support. Median $H$-score was 208 (probability of HLH according to the score, 92.5\%), and all patients had at least 4 to 7 criteria of the modified 2009 HLH criteria (genetic testing and NK cell activity were not available; sCD25 was tested in one patient). Causes of HLH were multiples: autoimmune diseases in four, infection (bacterial or viral) in three, and idiopathic in two. In addition to tocilizumab ( $8 \mathrm{mg} / \mathrm{kg}$ once, intravenously), five patients received concomitant treatment with dexamethasone $(n=$ $4)$, cyclophosphamide $(n=2)$, or intravenous immunoglobulins $(n=1)$. Remission was observed in $8 / 9$ patients after tocilizumab (88.9\%) whereas one developed refractory HLH, also unresponsive to rescue therapy with etoposide. Ferritin progressively decreased over the first 2 weeks (Fig. 1). One patient relapsed during the hospitalization and successfully received etoposide, but she ultimately died from unrelated gut ischemia. No patient developed profound neutropenia $\left(<500 \mathrm{cells} / \mathrm{mm}^{3}\right)$, except one who had also received cyclophosphamide. During the hospitalization, four patients died (sepsis-related multi-organ failure $n=1$; refractory $\operatorname{HLH} n=1$; organ support limitation $n=2$ ). None developed HLH relapse beyond the current hospitalization. Cytomegalovirus prophylaxis was pursued at least 3 months in transplant recipients.

In critically ill patients with severe forms of $\mathrm{HLH}$, etoposide rapidly reverses cytokine storm and improves 
Table 1 Characteristics and outcomes of nine patients with hemophagocytic syndrome who received tocilizumab. $M$, male; $F$, female; CAPS, catastrophic antiphospholipid syndrome; TMA, thrombotic microangiopathy; PVB19, parvovirus B19; LGL, large granular lymphocyte leukemia; HLH, hemophagocytic lymphohistiocytosis; DXM, dexamethasone; CYC, cyclophosphamide; IVIg, intravenous immunoglobulins; AlHA, autoimmune hemolytic anemia; SCT, stem cell transplantation; MMF, mycofenolate mofetil; Cst, corticosteroids; CSA, ciclosporin-A; CR, complete response; IS, immunosuppressive regimen; $M V$, mechanical ventilation; RRT, renal replacement therapy; VD, vasopressive drugs; OSL, organ support limitations; mHLH2009, modified 2009 HLH criteria

\begin{tabular}{|c|c|c|c|c|c|c|c|c|c|c|c|}
\hline & Age & Gender & Cause of HLH & $\begin{array}{l}\text { Underlying } \\
\text { immunodeficiency }\end{array}$ & $\begin{array}{l}\text { On-going IS at } \\
\text { the onset }\end{array}$ & $\begin{array}{l}\boldsymbol{H} \text {-score/ } \\
\text { mHLH2009 }\end{array}$ & $\begin{array}{l}\text { Other HLH } \\
\text { therapy }\end{array}$ & $\begin{array}{l}\text { Organ } \\
\text { supports }\end{array}$ & $\begin{array}{l}\mathrm{HLH} \\
\text { response }\end{array}$ & Relapse & Outcomes \\
\hline 1 & 59 & M & $\begin{array}{l}\text { Multiple } \\
\text { autoimmune } \\
\text { disorders }{ }^{\mathrm{a}} \text {, TMA }\end{array}$ & Cst & Cst & $\begin{array}{l}248 \\
(99.3 \%) / 7\end{array}$ & DXM, CYC & $\begin{array}{l}\text { MV, RRT, } \\
\text { VD }\end{array}$ & $C R$ & No & Alive \\
\hline 2 & 43 & M & Septicemia & Allogenic SCT & Cst & $\begin{array}{l}220 \\
(96.3 \%) / 5\end{array}$ & No & $\begin{array}{l}\text { MV, RRT, } \\
\text { VD }\end{array}$ & $C R$ & No & $\begin{array}{l}\text { Death (septic } \\
\text { shock; OSL) }\end{array}$ \\
\hline 3 & 23 & F & Idiopathic & $\begin{array}{l}\text { Heart } \\
\text { transplantation }\end{array}$ & $\begin{array}{l}\text { Tacrolimus, } \\
\text { MMF, Cst, IVlg }\end{array}$ & $\begin{array}{l}210(93 \%) / \\
5\end{array}$ & No & RRT, VD & $C R$ & No & Alive \\
\hline 4 & 60 & M & $\begin{array}{l}\text { Infections (varicella } \\
\text { zoster virus, } \\
\text { parvovirus B19, } \\
\text { HSV-2), septicemia }\end{array}$ & $\begin{array}{l}\text { Heart } \\
\text { transplantation }\end{array}$ & $\begin{array}{l}\text { Tacrolimus, } \\
\text { MMF, Cst }\end{array}$ & $\begin{array}{l}188(78 \%) / \\
5\end{array}$ & Etoposide & $\begin{array}{l}\text { MV, RRT, } \\
\text { VD }\end{array}$ & None & - & $\begin{array}{l}\text { Death (septic } \\
\text { shock, } \\
\text { aspergillosis, } \\
\text { refractory } \\
\text { HLH) }\end{array}$ \\
\hline 5 & 52 & M & $\begin{array}{l}\text { Parvovirus B19 and } \\
\text { CAPS }\end{array}$ & No & No & $\begin{array}{l}208 \\
(92.5 \%) / 5\end{array}$ & IVlg, DXM & $\begin{array}{l}\text { MV, RRT, } \\
\text { VD }\end{array}$ & $C R$ & No & Alive \\
\hline 6 & 53 & M & Idiopathic & $\begin{array}{l}\text { Liver } \\
\text { transplantation }\end{array}$ & $\begin{array}{l}\text { Tacrolimus, } \\
\text { MMF, Cst }\end{array}$ & $18(79 \%) / 5$ & No & MV, RRT & $C R$ & No & Alive \\
\hline 7 & 66 & $\mathrm{~F}$ & $\begin{array}{l}\text { Overlap syndrome, } \\
\text { TMA }\end{array}$ & Cst & Cst, rituximab & $\begin{array}{l}186 \\
(75.8 \%)\end{array}$ & DXM & $\begin{array}{l}\text { MV, RRT, } \\
\text { VD }\end{array}$ & $C R$ & $\begin{array}{l}\text { Yes } \\
\text { (etoposide) }\end{array}$ & $\begin{array}{l}\text { Death (gut } \\
\text { ischemia; } \\
\text { OSL) }\end{array}$ \\
\hline 8 & 57 & $\mathrm{~F}$ & Refractory AlHA & $\begin{array}{l}\text { T-LGL, B cell } \\
\text { lymphoma }\end{array}$ & Dxm, CsA, & $\begin{array}{l}188(78 \%) / \\
4\end{array}$ & CYC, DXM & $\begin{array}{l}\text { MV, RRT, } \\
\text { VD }\end{array}$ & $C R$ & No & $\begin{array}{l}\text { Death (septic } \\
\text { shock, } \\
\text { refractory } \\
\text { AlHA) }\end{array}$ \\
\hline 9 & 25 & $\mathrm{~F}$ & $\begin{array}{l}\text { S. hominis } \\
\text { bacteriemia, HSV-1 }\end{array}$ & $\begin{array}{l}\text { Kidney and liver } \\
\text { transplantation }\end{array}$ & $\begin{array}{l}\text { Tacrolimus, } \\
\text { MMF, Cst }\end{array}$ & $\begin{array}{l}218 \\
(95.8 \%) / 6\end{array}$ & No & - & $C R$ & No & Alive \\
\hline
\end{tabular}

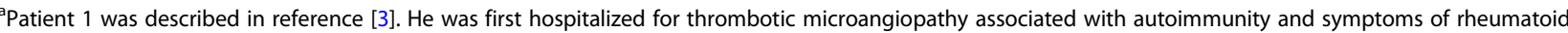
arthritis, anti-synthetase syndrome, systemic lupus erythematosus, cryoglobulinemia, and Sjogren syndrome

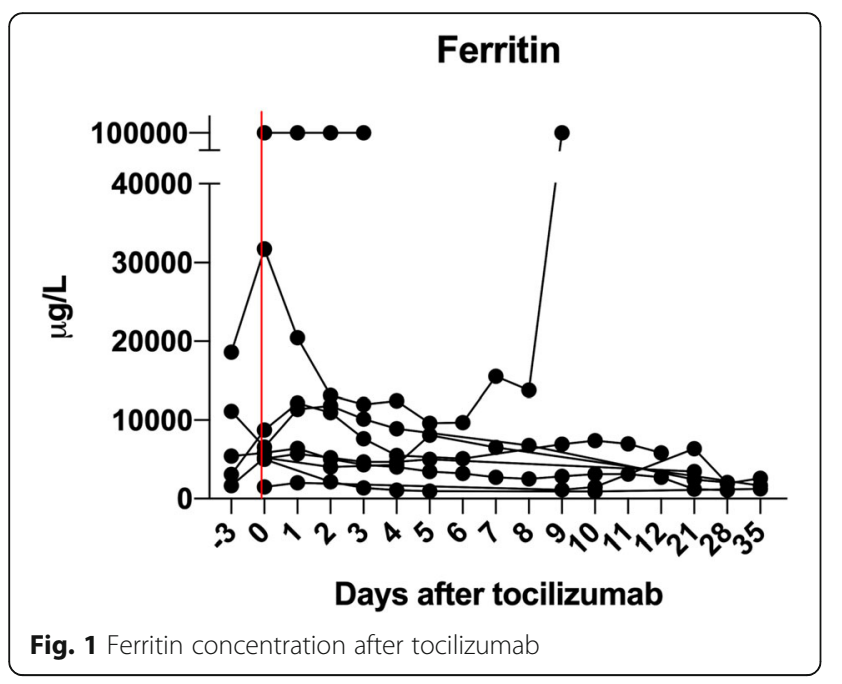

clinical condition [1]. HLH 94 and 2004 protocols were developed for children with primary HLH (50\% successes), but adult patients may be more at risk to develop chemotherapy toxicities [1]. Alternatives should thus be discussed in adult patients with chemotherapy-induced bone marrow failure, underlying autoimmune diseases requiring cytotoxic agents, or with a moderate form of HLH not related to hematological malignancies. In line with this need, the JAK $1 / 2$ inhibitor ruxolitinib was tested in a mouse model of genetic HLH. Its benefits were confirmed in patients with reactive HLH [5], but the oral administration may preclude its pharmacokinetic in critically ill patients requiring mechanical ventilation. Due to its intravenous administration, tocilizumab may thus be a valuable alternative after ruling out on-going bacterial or fungal sepsis.

In conclusion, IL-6-R blockade with tocilizumab may be an alternative in critically ill patients with moderate forms of HLH. Whether such beneficial effects may also be observed in the subset of patients with a cytokinerelated syndrome induced by the recently emerging SARS-CoV2 virus remains to be addressed. 


\section{Acknowledgements}

The authors thank the practitioners that followed the patients before and after their course in the ICU.

TAIDI (Toulouse Acquired Immune Deficiency and Infection) study group: Etienne Dufranc, Arnaud Del Bello, Julie Belliere, Nassim Kamar and Stanislas Faguer.

\section{Authors' contributions}

SF designed the study; ED collected the data; SF, NK, and ADB wrote the manuscript. All authors followed the patients. The authors read and approved the final manuscript.

\section{Funding}

None.

Availability of data and materials

The authors stated that all the data are available upon request.

Ethics approval and consent to participate

There are no ethical/legal conflicts involved in the article.

\section{Consent for publication}

Not applicable.

\section{Competing interests}

The authors have no conflicts of interest to disclose.

\section{Author details}

'Département de Néphrologie et Transplantation d'organes, Centre Hospitalier Universitaire de Toulouse, F-31000 Toulouse, France. ${ }^{2}$ Institut National de la Santé et de la Recherche Médicale, U1043, IFR-BMT, Hôpital Purpan, F-31000 Toulouse, France. ${ }^{3}$ Université Paul Sabatier - Toulouse 3, F-31000 Toulouse, France. ${ }^{4}$ Institut National de la Santé et de la Recherche Médicale, U1048, Institut des Maladies Métaboliques et Cardiovasculaires, Hôpital Rangueil, F-31000 Toulouse, France.

Received: 30 March 2020 Accepted: 6 April 2020

Published online: 22 April 2020

\section{References}

1. La Rosée P, Horne AC, Hines M, Greenwood TVB, Machowicz R, Berliner N, et al. Recommendations for the management of hemophagocytic lymphohistiocytosis in adults. Blood Ame Soc Hematol. 2019;133:2465-77.

2. Fardet L, Galicier L, Lambotte O, Marzac C, Aumont C, Chahwan D, et al. Development and validation of the HScore, a score for the diagnosis of reactive hemophagocytic syndrome. Arthritis Rheumatol. 2014;66:2613-20.

3. Fitzgerald JC, Weiss SL, Maude SL, Barrett DM, Lacey SF, Melenhorst JJ, et al. Cytokine release syndrome after chimeric antigen receptor T cell therapy for acute lymphoblastic leukemia. Crit Care Med. 2017;45:e124-5.

4. Faguer S, Vergez F, Peres M, Ferrandiz I, Casemayou A, Belliere J, et al. Tocilizumab added to conventional therapy reverses both the cytokine profile and CD8+Granzyme+ T-cells/NK cells expansion in refractory hemophagocytic lymphohistiocytosis. Hematol Oncol. 2016;34(1):55-7.

5. Ahmed A, Merrill SA, Alsawah F, Bockenstedt P, Campagnaro E, Devata S, et al. Ruxolitinib in adult patients with secondary haemophagocytic lymphohistiocytosis: an open-label, single-centre, pilot trial. Lancet Haematol. 2019;6:e630-7.

\section{Publisher's Note}

Springer Nature remains neutral with regard to jurisdictional claims in published maps and institutional affiliations.

Ready to submit your research? Choose BMC and benefit from:

- fast, convenient online submission

- thorough peer review by experienced researchers in your field

- rapid publication on acceptance

- support for research data, including large and complex data types

- gold Open Access which fosters wider collaboration and increased citations

- maximum visibility for your research: over $100 \mathrm{M}$ website views per year

At BMC, research is always in progress.

Learn more biomedcentral.com/submissions 